PROCEEDINGS OF THE

AMERICAN MATHEMATICAL SOCIETY

Volume 132, Number 11, Pages 3243-3250

S 0002-9939(04)07546-X

Article electronically published on June 17, 2004

\title{
MÖBIUS FUNCTION OF COORDINATE HYPERPLANES IN COMPLEX ELLIPSOIDS
}

\author{
WITOLD JARNICKI \\ (Communicated by Mei-Chi Shaw)
}

\begin{abstract}
For $p_{1}, \ldots, p_{n}>0$, let $\mathbb{E}=\left\{z \in \mathbb{C}^{n}: \sum_{j=1}^{n}\left|z_{j}\right|^{2 p_{j}}<1\right\}$ be a complex ellipsoid. We present effective formulas for the generalized Möbius and Green functions $m_{\mathbb{E}}(A, \cdot), g_{\mathbb{E}}(A, \cdot)$ in the case where $A:=\{z \in \mathbb{E}$ : $\left.z_{1} \cdots z_{k}=0\right\}(1 \leq k \leq n)$.
\end{abstract}

\section{INTRODUCTION AND MAIN RESULTS}

Let $G \subset \mathbb{C}^{n}$ be a domain, and let $A \subset G$. Define the generalized Green function

$$
\begin{aligned}
g_{G}(A, z):=\sup \{u(z): & u: G \longrightarrow[0,1), \log u \in \mathcal{P S H}(G), \\
& \left.\forall_{a \in A} \exists_{C=C(u, a)>0} \forall_{w \in G}: u(w) \leq C\|w-a\|\right\}, \quad z \in G,
\end{aligned}
$$

and the generalized Möbius function

$$
m_{G}(A, z):=\sup \left\{|f(z)|: f \in \mathcal{O}(G, \mathbb{D}),\left.f\right|_{A} \equiv 0\right\}, \quad z \in G,
$$

where $\mathbb{D}$ denotes the unit disc (cf. [Jar-Jar-Pfl 2003]).

The generalized Möbius and Green functions were recently studied by many authors (e.g. Lár-Sig 1998). Despite various proven properties, effective formulas for $m_{G}(A, \cdot)$ and $g_{G}(A, \cdot)$ are known only in a few special cases. An effective formula for the generalized Green function in the case of the Euclidean ball $G=\mathbb{B}_{2} \subset \mathbb{C}^{2}$ and the union of coordinate lines $A=\left\{(z, w) \in \mathbb{B}_{2}: z w=0\right\}$ was given in Ngu 2003.

Let $n \geq 2$. Let $p_{1}, \ldots, p_{n}>0$. Define the complex ellipsoid with exponents $p_{1}, \ldots, p_{n}$ :

$$
\mathbb{E}=\mathbb{E}_{p_{1}, \ldots, p_{n}}:=\left\{z \in \mathbb{C}^{n}: \sum_{j=1}^{n}\left|z_{j}\right|^{2 p_{j}}<1\right\} .
$$

Observe that $\mathbb{E}_{p_{1}, \ldots, p_{n}}$ is convex if and only if $p_{1}, \ldots, p_{n} \geq 1 / 2$ (cf. Jar-Pfl 1993, $\S 8.4)$.

For $G \subset \mathbb{C}^{n}, k \in\{1, \ldots, n\}$, we consider the sets

$$
A=A_{G, k}:=\left\{z \in G: z_{1} \cdots z_{k}=0\right\} .
$$

We give an effective formula for $g_{\mathbb{E}}\left(A_{\mathbb{E}, k}, \cdot\right)$ for any complex ellipsoid $\mathbb{E} \subset \mathbb{C}^{n}$ and any $k \in\{1, \ldots, n\}$ (Theorem $1.2(\mathrm{a})$ ). This is a generalization of a result in Ngu 2003 (with a much simpler proof). We prove that the $m_{\mathbb{E}}\left(A_{\mathbb{E}, k}, \cdot\right) \equiv$

Received by the editors June 23, 2003.

2000 Mathematics Subject Classification. Primary 32F45, 32 U35.

The author was supported in part by KBN grant no. 2 P03A 01522.

(C)2004 American Mathematical Society 
$g_{\mathbb{E}}\left(A_{\mathbb{E}, k}, \cdot\right)$ for the cases where $\mathbb{E}$ is convex (Theorem $1.2(\mathrm{~b})$ ) or where $k=1$, $n=2, p_{2} \geq 1 / 2$ (Theorem [1.2(c)). This is not true in the general nonconvex case; we prove that $m_{\mathbb{E}}\left(A_{\mathbb{E}, k}, \cdot\right) \not \equiv g_{\mathbb{E}}\left(A_{\mathbb{E}, k}, \cdot\right)$ if there exists a $j \in\{k+1, \ldots, n\}$ such that $p_{j}<1 / 2$ (Theorem $1.2(\mathrm{~d})$ ). Partial results for the case $k=n=2$ are given (Theorem 1.2(e)).

Definition 1.1. Let $p_{1}, \ldots, p_{n}>0,1 \leq k \leq n$. Put $\mathbb{E}:=\mathbb{E}_{p_{1}, \ldots, p_{n}}, A:=A_{\mathbb{E}, k}$. Let $z \in \mathbb{E}$ be such that the sequence $\left(p_{j}\left|z_{j}\right|^{2 p_{j}}\right)_{j=1}^{k}$ is monotonically increasing. For $s \in\{1, \ldots, k\}$ define

$$
q_{s}:=\sum_{j=1}^{s}\left(2 p_{j}\right)^{-1}, \quad r_{s}(z):=1-\sum_{j=s+1}^{n}\left|z_{j}\right|^{2 p_{j}}, \quad c_{s}(z):=r_{s}(z) / q_{s} .
$$

Let $d:=\max \left\{s \in\{1, \ldots, k\}: 2 p_{s}\left|z_{s}\right|^{2 p_{s}} \leq c_{s}(z)\right\}$. Define

$$
R_{\mathbb{E}}(A, z)=\prod_{j=1}^{d}\left|z_{j}\right|\left(\frac{2 p_{j}}{c_{d}(z)}\right)^{\frac{1}{2 p_{j}}} .
$$

The main results of the paper are the following.

Theorem 1.2. Under the above assumptions we have:

(a) $g_{\mathbb{E}}(A, \cdot) \equiv R_{\mathbb{E}}(A, \cdot)$,

(b) $m_{\mathbb{E}}(A, \cdot) \equiv g_{\mathbb{E}}(A, \cdot) \equiv R_{\mathbb{E}}(A, \cdot)$, for $p_{j} \geq 1 / 2, j=1, \ldots, n$,

(c) $m_{\mathbb{E}}(A, \cdot) \equiv g_{\mathbb{E}}(A, \cdot) \equiv R_{\mathbb{E}}(A, \cdot)$, for $k=1, n=2, p_{2} \geq 1 / 2$,

(d) $m_{\mathbb{E}}(A, \cdot) \not \equiv g_{\mathbb{E}}(A, \cdot)$ if there exists a $j \in\{k+1, \ldots, n\}$ with $p_{j}<1 / 2$,

(e) $m_{\mathbb{E}}(A, \cdot) \equiv g_{\mathbb{E}}(A, \cdot) \equiv R_{\mathbb{E}}(A, \cdot)$, for $k=n=2, p_{1} \leq p_{2}$, and either $p_{2} \geq 1 / 2$ or $8 p_{1}+4 p_{2}\left(1-p_{2}\right)>1$.

Observe that one can obtain the monotonicity of $\left(p_{j}\left|z_{j}\right|^{2 p_{j}}\right)_{j=1}^{k}$ for arbitrary $z \in \mathbb{E}$ by permutation of coordinates. The final result is a subdivision of $\mathbb{E}$ into $2^{k}-1$ subsets, each with $R_{\mathbb{E}}(A, \cdot)$ given by a formula of type $(*)$.

Remark 1.3. In the case where $p_{1}=\cdots=p_{n}=1$, the domain $\mathbb{E}$ is the Euclidean ball $\mathbb{B}_{n}$. Theorem 1.2 may then be formulated in the following simpler way.

Corollary 1.4. Assume that $\left|z_{1}\right| \leq \cdots \leq\left|z_{k}\right|$, and let

$$
d:=\max \left\{s \in\{1, \ldots, k\}: s\left|z_{s}\right|^{2}+\sum_{j=s+1}^{n}\left|z_{j}\right|^{2} \leq 1\right\} .
$$

Then

$$
m_{\mathbb{B}_{n}}(A, z)=g_{\mathbb{B}_{n}}(A, z)=\left(\frac{d}{1-\sum_{j=d+1}^{n}\left|z_{j}\right|^{2}}\right)^{\frac{d}{2}} \prod_{j=1}^{d}\left|z_{j}\right| .
$$

Remark 1.5. It is unknown to the author whether $m_{\mathbb{E}}(A, \cdot) \equiv g_{\mathbb{E}}(A, \cdot)$ for any $p_{j}>0, j=1, \ldots, k, p_{j} \geq 1 / 2, j=k+1, \ldots, n$. However, Theorem [1.2(e) and the author's research (cf. Remark 4.4) indicate that this is true at least in the case $k=n=2$.

Remark 1.6. Take $p_{j, \ell} \nearrow+\infty, j=1, \ldots, n$. Then

$$
G_{\ell}:=\mathbb{E}_{p_{1, \ell}, \ldots, p_{n, \ell}} \nearrow \mathbb{D}^{n} .
$$

Consequently,

$$
m_{G_{\ell}}\left(A_{G_{\ell}, k}, \cdot\right)=g_{G_{\ell}}\left(A_{G_{\ell}, k}, \cdot\right) \searrow m_{\mathbb{D}^{n}}\left(A_{\mathbb{D}^{n}, k}, \cdot\right)=g_{\mathbb{D}^{n}}\left(A_{\mathbb{D}^{n}, k}, \cdot\right)
$$


(cf. [Jar-Jar-Pfl 2003], Property 2.7) and hence

$$
m_{\mathbb{D}^{n}}\left(A_{\mathbb{D}^{n}, k}, z\right)=\left|z_{1}\right| \cdots\left|z_{k}\right|, \quad z \in \mathbb{D}^{n} .
$$

However, we need the formula for $g_{\mathbb{D}^{n}}\left(A_{\mathbb{D}^{n}, k}, \cdot\right)$ before we prove Theorem 1.2 (cf. Lemma 2.11).

\section{Proof of Theorem 1.2}

Lemma 2.1. Let $n \in \mathbb{N}, 1 \leq k \leq n$. Then

$$
m_{\mathbb{D}^{n}}\left(A_{\mathbb{D}^{n}, k}, z\right)=g_{\mathbb{D}^{n}}\left(A_{\mathbb{D}^{n}, k}, z\right)=\left|z_{1}\right| \cdots\left|z_{k}\right|, \quad z \in \mathbb{D}^{n} .
$$

Proof of Lemma 2.1 (due to P. Pflug). Obviously,

$$
\left|z_{1}\right| \cdots\left|z_{k}\right| \leq m_{\mathbb{D}^{n}}\left(A_{\mathbb{D}^{n}, k}, z\right) \leq g_{\mathbb{D}^{n}}\left(A_{\mathbb{D}^{n}, k}, z\right) .
$$

It remains to prove that $g_{\mathbb{D}^{n}}\left(A_{\mathbb{D}^{n}, k}, z\right) \leq\left|z_{1}\right| \cdots\left|z_{k}\right|$.

It is enough to prove that for any $1 \leq k \leq n$ and for any function $u: \mathbb{D}^{n} \longrightarrow[0,1)$ such that $\log u \in \mathcal{P S H}\left(\mathbb{D}^{n}\right)$ and $u(z) \leq C(a)\|z-a\|, a \in A_{\mathbb{D}^{n}, k}, z \in \mathbb{D}^{n}$, we have $u(z) \leq\left|z_{1}\right| \cdots\left|z_{k}\right|$. We proceed by induction on $k$.

For $k=1$ the inequality follows from the Schwarz-type lemma for logarithmically subharmonic functions $u\left(\cdot, z_{2}, \ldots, z_{n}\right), z_{2}, \ldots, z_{n} \in \mathbb{D}$.

For $k>1$ we first apply the case $k=1$ and get $u\left(z_{1}, \ldots, z_{n}\right) \leq\left|z_{1}\right|, z \in \mathbb{D}^{n}$. Applying the inductive assumption to $u\left(z_{1}, \cdot\right) /\left|z_{1}\right|, z_{1} \in \mathbb{D}$, finishes the proof.

Take a $u: \mathbb{E} \longrightarrow[0,1)$ with $\log u \in \mathcal{P S H}(\mathbb{E})$ and $u(\zeta) \leq C(a)\|\zeta-a\|, a \in A$, $\zeta \in \mathbb{E}$. Consider the mapping

$$
\iota_{z}: \mathbb{D}^{d} \ni\left(\zeta_{1}, \ldots, \zeta_{d}\right) \longmapsto\left(\zeta_{1}\left(\frac{c_{d}(z)}{2 p_{1}}\right)^{\frac{1}{2 p_{1}}}, \ldots, \zeta_{d}\left(\frac{c_{d}(z)}{2 p_{d}}\right)^{\frac{1}{2 p_{d}}}, z_{d+1}, \ldots, z_{n}\right) \in \mathbb{E} .
$$

Applying the holomorphic contractivity of the generalized Green function and Lemma 2.1] proves that $m_{\mathbb{E}}(A, z) \leq g_{\mathbb{E}}(A, z) \leq R_{\mathbb{E}}(A, z)$.

It remains to prove that $m_{\mathbb{E}}(A, z) \geq R_{\mathbb{E}}(A, z)\left(\right.$ resp. $\left.g_{\mathbb{E}}(A, z) \geq R_{\mathbb{E}}(A, z)\right)$. In the case $d=k=n$ it suffices to take

$$
f(\zeta):=\prod_{j=1}^{d} \zeta_{j}\left(\frac{2 p_{j}}{c_{d}(z)}\right)^{\frac{1}{2 p_{j}}} .
$$

Then $f \in \mathcal{O}(\mathbb{E}, \mathbb{D})$ and $|f(z)|=R_{\mathbb{E}}(A, z)$.

It remains to prove Theorem 1.2 in the remaining cases. We may assume that $z_{j} \neq 0, j=1, \ldots, d$. Consider the following lemma.

Lemma 2.2. Define $\mathbb{E}^{\prime}:=\mathbb{E}_{p_{d+1}, \ldots, p_{n}}$.

(a) Let $v: \mathbb{E}^{\prime} \longrightarrow[0,1), v \not \equiv 0$ be such that $\log v \in \mathcal{P S H}\left(\mathbb{E}^{\prime}\right)$ and $v(\zeta) \leq\left|\zeta_{j}\right|$, $\zeta \in \mathbb{E}^{\prime}, j=d+1, \ldots, k$, and the mapping

$$
\mathbb{E}^{\prime} \ni\left(\zeta_{d+1}, \ldots, \zeta_{n}\right) \longmapsto v\left(\zeta_{d+1}, \ldots, \zeta_{n}\right) r_{d}^{q_{d}}(\zeta)
$$

attains its maximum value $M$ at $\left(z_{d+1}, \ldots, z_{n}\right)$. Then $g_{\mathbb{E}}(A, z)=R_{\mathbb{E}}(A, z)$.

(b) The following conditions are equivalent:

- There exists an $h \in \mathcal{O}\left(\mathbb{E}^{\prime}\right), h \not \equiv 0$ such that $h(\zeta)=0$ for $\zeta_{d+1} \cdots \zeta_{k}=0$ and the mapping

$$
\mathbb{E}^{\prime} \ni\left(\zeta_{d+1}, \ldots, \zeta_{n}\right) \longmapsto\left|h\left(\zeta_{d+1}, \ldots, \zeta_{n}\right)\right| r_{d}^{q_{d}}(\zeta)
$$

attains its maximum value $M$ at the point $\left(z_{d+1}, \ldots, z_{n}\right)$,

- $m_{\mathbb{E}}(A, z)=R_{\mathbb{E}}(A, z)$. 
We present a proof in Section [3, The result above reduces the proof of Theorem 1.2 to the following propositions.

Proposition 2.3. There exists a $v$ as required in Lemma 2.2(a).

Proposition 2.4. Using the notation of Theorem 1.2, assume that $p_{j} \geq 1 / 2$, $j=d+1, \ldots, n$. Then there exists an $h \in \mathcal{O}\left(\mathbb{E}^{\prime}\right)$ as required in Lemma 2.2(b).

Proposition 2.5. Using the notation of Theorem 1.2, assume that $p_{k+1}<1 / 2$. Then one cannot find an $h$ as required in Lemma 2.2(b) for $\left|z_{\ell}\right| \neq 0$ small enough, $\ell=1, \ldots, k+1$ and $z_{\ell}=0, \ell=k+2, \ldots, n$.

Proposition 2.6. Using the notation of Theorem 1.2, assume that $n=k=2$. Additionally, assume that $p_{2} \geq 1 / 2$ or $8 p_{1}+4 p_{2}\left(1-p_{2}\right)>1$. Then there exists an $h \in \mathcal{O}(\mathbb{D})$ as required in Lemma 2.2(b).

\section{Proof of Lemma 2.2}

(a) Put

$$
u\left(\zeta_{1}, \ldots, \zeta_{n}\right):=M^{-1}\left(\prod_{j=1}^{d}\left(2 p_{j}\right)^{\frac{1}{2 p_{j}}}\left|\zeta_{j}\right|\right) q_{d}^{q_{d}} v\left(\zeta_{d+1}, \ldots, \zeta_{n}\right) .
$$

Obviously $\log u \in \mathcal{P S H}(\mathbb{E})$ and $u(\zeta) \leq C\left|\zeta_{j}\right| \leq C\|\zeta-a\|, \zeta \in \mathbb{E}$, whenever $a_{j}=0$ for some $j \in\{1, \ldots, k\}$. For $\zeta \in \mathbb{E}$ we have

$$
\left.u(\zeta) \leq M^{-1}\left(\frac{\sum_{j=1}^{d}\left|\zeta_{j}\right|^{2 p_{j}}}{q_{d}}\right)^{q_{d}} q_{d}^{q_{d}} v\left(\zeta_{d+1}, \ldots, \zeta_{n}\right)<1\right]
$$

Consequently $u: \mathbb{E} \longrightarrow[0,1)$. On the other hand,

$$
u(z)=M^{-1} R_{\mathbb{E}}(A, z) r_{d}^{q_{d}}(z) v\left(z_{d+1}, \ldots, z_{n}\right)=R_{\mathbb{E}}(A, z) .
$$

(b) Assume that such an $h$ exists. Put

$$
f\left(\zeta_{1}, \ldots, \zeta_{n}\right):=M^{-1}\left(\prod_{j=1}^{d}\left(2 p_{j}\right)^{\frac{1}{2 p_{j}}} \zeta_{j}\right) q_{d}^{q_{d}} h\left(\zeta_{d+1}, \ldots, \zeta_{n}\right) .
$$

Observe that $f(\zeta)=0$ for $\zeta \in A$. Similarly as in (a) we prove that $|f(\zeta)|<1, \zeta \in \mathbb{E}$ and $f(z)=R_{\mathbb{E}}(A, z)$.

Assume now that $m_{\mathbb{E}}(A, z)=R_{\mathbb{E}}(A, z)$. Let $f \in \mathcal{O}(\mathbb{E}, \mathbb{D})$ be such that $\left.f\right|_{A} \equiv 0$ and $|f(z)|=R_{\mathbb{E}}(A, z)$ (cf. [Jar-Jar-Pfl 2003, Property 2.5). Put

$$
h(\zeta):=\frac{\partial^{d} f}{\partial z_{1} \ldots \partial z_{d}}(0, \zeta) .
$$

\footnotetext{
${ }^{1}$ Let $a_{1}, \ldots, a_{d} \geq 0, w_{1}, \ldots, w_{d}>0$. Then$$
\prod_{j=1}^{d} a_{j}^{w_{j}} \leq\left(\frac{\sum_{j=1}^{d} w_{j} a_{j}}{\sum_{j=1}^{d} w_{j}}\right)^{\sum_{j=1}^{d} w_{j}} .
$$ 
By definition, we have $h(\zeta)=0$ for $\zeta_{d+1} \cdots \zeta_{k}=0$. Applying the Schwarz lemma to the mapping $f \circ \iota_{\zeta}, \zeta \in \mathbb{E}^{\prime}$, we get

$$
\begin{aligned}
& \left|h\left(\zeta_{d+1}, \ldots, \zeta_{n}\right)\right| r_{d}^{q_{d}}(\zeta) \leq\left(\prod_{j=1}^{d}\left(2 p_{j}\right)^{\frac{1}{2 p_{j}}}\right) q_{d}^{q_{d}}, \quad \zeta \in \mathbb{E}^{\prime}, \\
& \left|h\left(z_{d+1}, \ldots, z_{n}\right)\right| r_{d}^{q_{d}}(z)=\left(\prod_{j=1}^{d}\left(2 p_{j}\right)^{\frac{1}{2 p_{j}}}\right) q_{d}^{q_{d}} .
\end{aligned}
$$

4. Proof of Propositions 2.3, 2.4, 2.5, and 2.6

Proof of Proposition 2.3. We may assume that $z_{d+1}, \ldots, z_{n} \geq 0$. Consider functions of the form

$$
v_{\alpha}\left(\zeta_{d+1}, \ldots, \zeta_{n}\right)=\left(\prod_{j=d+1}^{k}\left|\zeta_{j}\right|^{1+\alpha_{j}}\right)\left(\prod_{j=k+1}^{n}\left|\zeta_{j}\right|^{\alpha_{j}}\right),
$$

where $\alpha=\left(\alpha_{d+1}, \ldots, \alpha_{n}\right), \alpha_{d+1}, \ldots, \alpha_{n} \geq 0$. Obviously $v: \mathbb{E}^{\prime} \longrightarrow[0,1), \log v \in$ $\mathcal{P S H}\left(\mathbb{E}^{\prime}\right)$, and $v(\zeta) \leq\left|\zeta_{j}\right| \leq\|\zeta-a\|, \zeta \in \mathbb{E}^{\prime}$, whenever $a_{j}=0$ for some $j \in$ $\{d+1, \ldots, k\}$. Since $v_{\alpha}\left(\zeta_{d+1}, \ldots, \zeta_{n}\right)=v_{\alpha}\left(\left|\zeta_{d+1}\right|, \ldots,\left|\zeta_{n}\right|\right)$, it is enough to find an $\alpha$ such that the function

$$
\mathbb{E}^{\prime} \cap \mathbb{R}_{+}^{n-d} \ni\left(t_{d+1}, \ldots, t_{n}\right) \longmapsto v_{\alpha}\left(t_{d+1}, \ldots, t_{n}\right) r_{d}^{q_{d}}(t)
$$

attains its maximum at $\left(z_{d+1}, \ldots, z_{n}\right)$. Considering the partial (logarithmic) derivatives results in the following equations:

$$
\begin{array}{r}
0=1+\alpha_{j}-2 p_{j} q_{d} \frac{t_{j}^{2 p_{j}}}{r_{d}(t)}, \quad j=d+1, \ldots, k, \\
0=\alpha_{j}-2 p_{j} q_{d} \frac{t_{j}^{2 p_{j}}}{r_{d}(t)}, \quad j=k+1, \ldots, n .
\end{array}
$$

These give formulas for $\alpha_{d+1}, \ldots, \alpha_{n}$ such that $\left(z_{d+1}, \ldots, z_{n}\right)$ is the common zero of the derivatives. To prove that there are no other points like this, consider a reformulation of the above equations:

$$
\begin{aligned}
& r_{d}(t)=\frac{2 p_{j} q_{d} t_{j}^{2 p_{j}}}{1+\alpha_{j}}, \quad j=d+1, \ldots, k, \\
& r_{d}(t)=\frac{2 p_{j} q_{d} t_{j}^{2 p_{j}}}{\alpha_{j}}, \quad j=k+1, \ldots, n .
\end{aligned}
$$

The left side is decreasing in any of the variables, while the right sides are increasing. Thus, at most one common zero is allowed.

It remains to check whether $\alpha_{j} \geq 0, j=d+1, \ldots, n$. Obviously, this is true for $j=k+1, \ldots, n$ and in the remaining cases we have

$$
\alpha_{j}=\frac{2 p_{j} q_{d} t_{j}^{2 p_{j}}-r_{d}(t)}{t_{j} r_{d}(t)} \geq 0
$$

since this is the way we have chosen $d$. 
Proof of Proposition 2.4. We may assume that $z_{d+1}, \ldots, z_{n} \geq 0$. Consider functions of the form

$$
h_{\alpha}\left(\zeta_{d+1}, \ldots, \zeta_{n}\right)=\left(\prod_{j=d+1}^{k} \zeta_{j} e^{\alpha_{j} \zeta_{j}}\right)\left(\prod_{j=k+1}^{n} e^{\alpha_{j} \zeta_{j}}\right),
$$

where $\alpha=\left(\alpha_{d+1}, \ldots, \alpha_{n}\right), \alpha_{d+1}, \ldots, \alpha_{n} \geq 0$.

Since $\left|h_{\alpha}\left(\zeta_{d+1}, \ldots, \zeta_{n}\right)\right| \leq h_{\alpha}\left(\left|\zeta_{d+1}\right|, \ldots,\left|\zeta_{n}\right|\right)$, it is enough to find an $\alpha$ such that

$$
\mathbb{E}^{\prime} \cap \mathbb{R}_{+}^{n-d} \ni\left(t_{d+1}, \ldots, t_{n}\right) \longmapsto h_{\alpha}\left(t_{d+1}, \ldots, t_{n}\right) r_{d}^{q_{d}}(t)
$$

attains its maximum at $\left(z_{d+1}, \ldots, z_{n}\right)$. Considering the partial (logarithmic) derivatives results in the following equations:

$$
\begin{array}{r}
0=\frac{1}{t_{j}}+\alpha_{j}-2 p_{j} q_{d} \frac{t_{j}^{2 p_{j}-1}}{r_{d}(t)}, \quad j=d+1, \ldots, k, \\
0=\alpha_{j}-2 p_{j} q_{d} \frac{t_{j}^{2 p_{j}-1}}{r_{d}(t)}, \quad j=k+1, \ldots, n .
\end{array}
$$

We continue as in the proof of Proposition 2.3.

Proof of Proposition 2.5. Consider the following two lemmas.

Lemma 4.1. Assume that there exist $0<c<b<1$ such that the function

$$
\varphi:[0, b] \ni t \longmapsto\left(1-t^{p}\right)^{-q}
$$

is strictly concave and

$$
\varphi(0)+\frac{b}{c}(\varphi(c)-\varphi(0))>\varphi(b)+2 .
$$

Let $f \in \mathcal{O}(\mathbb{D}), f \not \equiv 0$ such that $|f(\zeta)| / \varphi(|\zeta|)$ attains its maximum at $w_{0}$. Then $w_{0}=0$ or $\left|w_{0}\right| \geq c$.

Proof. Assume that $\left|w_{0}\right| \in(0, c)$. We may assume that $\left|f\left(w_{0}\right)\right|=\varphi\left(\left|w_{0}\right|\right)$. Consider the function

$$
\psi:[0, b] \ni t \longmapsto|f(0)|+\frac{t}{\left|w_{0}\right|}\left|f\left(w_{0}\right)-f(0)\right| .
$$

From $\psi(0) \leq \varphi(0), \psi\left(\left|w_{0}\right|\right) \geq \varphi\left(\left|w_{0}\right|\right)$, and the convexity condition we get

$$
\begin{aligned}
\psi(b)=|f(0)|+\frac{b}{\left|w_{0}\right|}\left|f\left(w_{0}\right)-f(0)\right| & \geq \varphi(0)+\frac{b}{\left|w_{0}\right|}\left|\varphi\left(\left|w_{0}\right|\right)-\varphi(0)\right| \\
& \geq \varphi(0)+\frac{b}{c}|\varphi(c)-\varphi(0)|>\varphi(b)+2 .
\end{aligned}
$$

The Schwarz lemma and the maximum principle imply that there exists a $w \in \mathbb{D}$ with $|w|=b$ and

$$
\frac{|f(w)-f(0)|}{|w|} \geq \frac{\left|f\left(w_{0}\right)-f(0)\right|}{\left|w_{0}\right|} .
$$

This means that

$$
\begin{aligned}
|f(w)| & \geq|f(w)-f(0)|-|f(0)|=|f(0)|+|f(w)-f(0)|-2|f(0)| \\
& \geq \psi(b)-2|f(0)|>\varphi(b)+2-2|f(0)| \geq \varphi(b)=\varphi(|w|) .
\end{aligned}
$$

This contradicts the maximality of $|f(\zeta)| / \varphi(|\zeta|)$ at $w_{0}$. 
Lemma 4.2. Assume that $p \in(0,1), q>0$. Then there exist $b \in(0,1), c \in(0, b)$ as required in Lemma 4.1.

Proof. We have

$$
\varphi^{\prime}(t)=\frac{p q}{t^{1-p}\left(1-t^{p}\right)^{q+1}}
$$

which is decreasing for small $t$. To prove the existence of $c$ observe that

$$
\lim _{d \longrightarrow 0^{+}} \frac{\varphi(d)-\varphi(0)}{d}=+\infty .
$$

Observe that $d=k$ for $\left|z_{\ell}\right|$ small enough, $\ell=1, \ldots, k$. Let $h \in \mathcal{O}\left(\mathbb{E}^{\prime}\right)$ and consider $f(\zeta):=h(\zeta, 0, \ldots, 0), \zeta \in \mathbb{D}$. Put $p:=2 p_{k+1}, q=q_{d}$. Let $b, c$ be as in Lemma 4.2. It follows from Lemma 4.1 that $0 \neq\left|w_{0}\right|<c$ cannot be a maximum of $\mathbb{D} \ni \zeta \longmapsto|f(\zeta)| / \varphi(|\zeta|)$. In particular, $z$ cannot be a maximum of $\mathbb{E}^{\prime} \ni \zeta \longmapsto|h(\zeta)| r_{d}^{q_{d}}(\zeta)$ for $0 \neq\left|z_{k+1}\right|<c$.

Proof of Proposition 2.6. Consider the following lemma.

Lemma 4.3. Let $a, c>0, t_{0} \in(0,1)$ be such that $c \geq 1$ or $4 a+2 c>1+c^{2}$, $t_{0}^{c}>\tau:=a /(a+c)$. Then there exist $b>0$ and $r \geq 1$ such that

$$
h:[0,1] \ni t \longmapsto \frac{t^{a}}{(r-t)^{b}}\left(1-t^{c}\right) \in[0,1]
$$

admits its maximum at $t_{0}$.

Proof. Comparing $\varphi:=t h^{\prime}(t) / h(t)$ to zero we get the equation

$$
\varphi(r, t)=a+\frac{b t}{r-t}-\frac{c t^{c}}{1-t^{c}}=0 .
$$

This gives us a formula for $r$ :

$$
r(t)=\frac{t\left(b-b t^{c}-a+a t^{c}+t^{c} c\right)}{-a+a t^{c}+t^{c} c} .
$$

Observe that

$$
\lim _{t \longrightarrow \tau^{1 / c}} r(t)=+\infty, \quad \lim _{t \longrightarrow 1} r(t)=1 .
$$

In order to prove that $r(t)>1$ and that $\varphi(r, \cdot)$ has only one zero it suffices to show that $r^{\prime}(t)<0$. We have

$$
r^{\prime}(t)=\frac{\left(-(a+c) t^{2 c}+\left(2 a+c-c^{2}\right) t^{c}-a\right) b+\left(a t^{c}+t^{c} c-a\right)^{2}}{\left(a t^{c}+t^{c} c-a\right)^{2}} .
$$

It remains to show that the coefficient $\alpha\left(t^{c}\right)$ next to $b$ is negative. We have

$$
\begin{aligned}
\alpha(\tau) & =\frac{-a c^{2}}{a+c}<0, \\
\alpha(1) & =-c^{2}<0, \\
\alpha^{\prime}(u) & =-2(a+c) u+2 a+c-c^{2} .
\end{aligned}
$$

Let $u_{0}$ be the zero of $\alpha^{\prime}(u)$. For $c \geq 1$ we have $u_{0} \leq \tau$ and we are done. Otherwise $u_{0} \in(\tau, 1)$ and $4(a+c) \alpha\left(u_{0}\right)=c^{2}\left(1+c^{2}-4 a-2 c\right)<0$. 
We may assume that $z_{0}>0$. Put $a=2 p_{1}, c=2 p_{2}, t_{0}=z_{0}$. Let $r$ be as in Lemma 4.3. Putting

completes the proof.

$$
h(\zeta)=\frac{\zeta}{(r-\zeta)^{b / a}}
$$

Remark 4.4. The calculations in the proof of Proposition 2.6 can be performed using alternative function families, e.g.,

$$
\begin{aligned}
h_{j}(\zeta) & :=(\zeta+r)^{k}, \quad r \geq 0, k=0,1,2, \ldots, \\
h_{j}(\zeta) & :=(\zeta+1)^{\alpha}, \quad \alpha>0, \\
h_{j}(\zeta) & :=(r-\zeta)^{\alpha} \zeta^{k}, \quad \alpha<0, r \geq 1, k=0,1,2, \ldots, \\
h_{j}(\zeta) & :=\left(\frac{\zeta+\delta}{1+\delta \zeta}\right) \zeta^{k}, \quad \delta \in[0,1], k=0,1,2, \ldots
\end{aligned}
$$

However, the author was unable to solve the general case using any of them.

\section{ACKNOWLEDGEMENT}

The author is indebted to professor Peter Pflug for his valuable comments on the paper.

\section{REFERENCES}

[Jar-Jar-Pfl 2003] M. Jarnicki, W. Jarnicki, P. Pflug, On extremal holomorphically contractible families, Ann. Polon. Math. 81 (2003), 183-199. MR 2004b:32016

[Jar-Pfl 1993] M. Jarnicki, P. Pflug, Invariant Distances and Metrics in Complex Analysis, de Gruyter Exp. Math. 9, de Gruyter, 1993. MR 94k:32039

[Lár-Sig 1998] F. Lárusson, R. Sigurdsson, Plurisubharmonic functions and analytic discs on manifolds, J. Reine Angew. Math. 501 (1998), 1-39. MR 99e:32020

[Ngu 2003] Nguyen Quang Dieu, Continuity of pluricomplex Green functions with poles along a hypersurface, preprint.

Jagiellonian University, Institute of Mathematics, Reymonta 4, 30-059 Kraków, POLAND

E-mail address: wmj@im.uj.edu.pl

Current address: Universität Osnabrück, Fachbereich Mathematik/Informatik, Albrechtstraße 28, 49069 Osnabrück, Germany 